\title{
Global Dynamics Analysis of Homogeneous New Products Diffusion Model
}

\author{
Shuping $\mathrm{Li}^{1,2}$ and Zhen $\mathrm{Jin}^{2,3}$ \\ ${ }^{1}$ School of Mechatronic Engineering, North University of China, Taiyuan 030051, China \\ ${ }^{2}$ Department of Mathematics, North University of China, Taiyuan 030051, China \\ ${ }^{3}$ Complex Systems Center, Shanxi University, Taiyuan 030006, China \\ Correspondence should be addressed to Zhen Jin; jinzhn@263.net
}

Received 4 June 2013; Revised 3 October 2013; Accepted 4 October 2013

Academic Editor: Sarangapani Jagannathan

Copyright (C) 2013 S. Li and Z. Jin. This is an open access article distributed under the Creative Commons Attribution License, which permits unrestricted use, distribution, and reproduction in any medium, provided the original work is properly cited.

A mathematical model with stage structures is presented that incorporates the awareness stage and the decision-making stage; individuals exchange product information by two channels: mass media and interpersonal communication. When the persuasive advertisement is neglected in the decision-making stage, we find a threshold value about whether new products diffusion is successful or not. When the persuasive advertisement is considered, there must exist a positive equilibrium under some parameter condition; moreover, it must be globally asymptotically stable as long as it exists. Results show that the persuasive advertisement in the decision-making stage does not influence new products' successful diffusion, but the critical value that new products diffuse successfully decreases when the persuasive advertisement is considered. Some numerical simulations confirm our theoretical analysis and demonstrate the influence of parameters on the system. Corresponding to the analysis results, some diffusion strategies are provided.

\section{Introduction}

New products diffusion is the process by which some new products information is communicated through certain channels over time among the members of a social system. It is a special type of communication in which the messages are concerned with new ideas. Previously we defined communication as the process by which participants create and share information with one another in order to reach a mutual understanding. The essence of the new products diffusion process is the product information exchanged by which one individual communicates a new product to one or several others. At its most elementary form, the process involves (1) a propagation about some new product by mass media, (2) an individual or other unit of adoption that is aware of, or experienced with, using some new product, (3) another individual or other unit that is not yet aware of this new product, and (4) a communication channel connecting the two units. The heart of new products diffusion process is that more people know and adopt new products; therefore, on one hand, mass media channels are all those means of transmitting new products information that involve a mass medium, such as radio, television, newspapers, and so on, which enable a source of one or a few individuals to reach an audience of many; on the other hand, most people depend mainly upon a subjective evaluation of an innovation that is conveyed to them from other individuals like themselves who have previously adopted the innovation. So, interpersonal channels are more effective in persuading an individual to adopt new products, especially if the interpersonal channel links two or more individuals who are near-peers.

The Bass model has become an important exemplar in marketing science. For over three decades, this model has been the main impetus underlying diffusion research and has been widely used to understand the spread of new products [1-3]. On the base of the Bass model, many dynamics models are used to study new products diffusion [4-7]. In fact, the consumer's aware formation of new products and decision making process on the new product adoption involves a complex interaction of various external and internal factors like mass media, advertising, word-ofmouth, personal preferences, and experience. Interpersonal 
communications, without a doubt, constitute an important communication media especially for social groups that are hardly reachable by mass media advertising [8-10]. In this paper, for the simplicity of mathematical analysis, we consider two stages in the diffusion process: the stage of awareness of information and the stage of decision making. Specifically, in the first stage, enterprises transfer new production information to consumers through the informative advertisement, or individuals transfer new product information to each other. So, individuals become aware of information and they are interested in the product and evaluate its value to adopt it or not to adopt it. In the decision-making stage, enterprises change consumers' preferences through the persuasive advertisement, or a friend's opinion or advice also influences individuals' preference, so individuals try or adopt the product in the second stage. In view of these, assuming that the product information is spread due to word-of-mouth among people's direct contacts and mass media channels, we propose a homogeneous model with two stages of new products diffusion.

The organization of this paper is as follows. In the next section, we set up a homogeneous model about new products diffusion and discuss its equilibriums and their stability when the persuasive advertisement is neglected or considered in the decision-making stage and, respectively, obtain conditions that the diffusion is successful; further, by comparing two critical values, we analyse the effect of the persuasive advertisement in the decision-making stage. In Section 3, theoretical results are confirmed by numerical simulations. In Section 4, we give sensitive analysis of parameters and the influence of parameters on the adopters by simulations when diffusion is successful. Finally, we give a brief conclusion and discussion in Section 5.

\section{Model}

In this section, incorporating an awareness stage and a decision-making stage into the Bass model, we put forward the model as follows with the standard incidence rate:

$$
\begin{gathered}
\frac{d U(t)}{d t}=-p U-\beta_{1} U \frac{I}{N}-\beta_{2} U \frac{A}{N}+\gamma I+\mu A, \\
\frac{d I(t)}{d t}=p U+\beta_{1} U \frac{I}{N}+\beta_{2} U \frac{A}{N}-\gamma I-\alpha I-\beta_{3} I \frac{A}{N}, \\
\frac{d A(t)}{d t}=\alpha I+\beta_{3} I \frac{A}{N}-\mu A .
\end{gathered}
$$

Here, $U(t)$ denote the number of those individuals who have not been aware of the product at time $t, I(t)$ denote the number of those individuals who have been aware of the information about the product but have not yet adopted it at time $t$, and $A(t)$ denote the number of those individuals who have adopted the product at time $t$. Consider that $N=$ $U(t)+I(t)+A(t)$, the total number of people, is a constant. All parameters are positive constants and summarized in Table 1. Note that who have been aware of the information about the product but have not yet adopted it also spread the information of the product, so we incorporate the item
TABle 1: Parameters of the model.

\begin{tabular}{ll}
\hline Parameters & Description \\
\hline$p$ & $\begin{array}{l}\text { The fractions of individuals who have not } \\
\text { been aware of the product obtain the new } \\
\text { product information from the } \\
\text { informative advertisement. }\end{array}$ \\
\hline$\beta_{1}$ & $\begin{array}{l}\text { The effective transmission coefficient } \\
\text { between community } U \text { and } I \text { in the } \\
\text { awareness stage. }\end{array}$ \\
\hline$\beta_{2}$ & $\begin{array}{l}\text { The effective transmission coefficient } \\
\text { between community } U \text { and } A \text { in the } \\
\text { awareness stage. }\end{array}$ \\
\hline$\beta_{3}$ & $\begin{array}{l}\text { The effective transmission coefficient } \\
\text { between community } I \text { and } A \text { in the } \\
\text { decision-making stage. }\end{array}$ \\
\hline$\gamma$ & $\begin{array}{l}\text { The rate at which individuals in } \\
\text { awareness class forget the information of } \\
\text { the product. }\end{array}$ \\
\hline$\mu$ & $\begin{array}{l}\text { The coefficient of discontinuance rate of } \\
\text { adopters. }\end{array}$ \\
\hline & $\begin{array}{l}\text { The fractions of individuals who have } \\
\text { been aware of the product turn to be } \\
\text { adopters owing to the persuasive } \\
\text { advertisement. }\end{array}$ \\
\hline
\end{tabular}

$\beta_{1} U(I / N)$ into our model which extends the earlier model [11]. When $\beta_{1}=0$, system (1) becomes the model in [11].

Mass media channels are considered a more effective way to generate awareness of the new product [12]; for this reason, we further assume that $p>\beta_{1}, p>\beta_{2}$. Denote the relative densities of $U(t), I(t)$, and $A(t)$ at time $t$ by $u(t), i(t)$, and $a(t)$, respectively; then, system (1) can be rewritten as

$$
\begin{gathered}
\frac{d u(t)}{d t}=-p u-\beta_{1} u i-\beta_{2} u a+\gamma i+\mu a, \\
\frac{d i(t)}{d t}=p u+\beta_{1} u i+\beta_{2} u a-\gamma i-\alpha i-\beta_{3} i a, \\
\frac{d a(t)}{d t}=\alpha i+\beta_{3} i a-\mu a .
\end{gathered}
$$

Noting that $u(t)+i(t)+a(t)=1$, system (2) is reduced to

$$
\begin{gathered}
\frac{d i(t)}{d t}=\left(p+\beta_{1} i+\beta_{2} a\right)(1-i-a)-\gamma i-\alpha i-\beta_{3} i a, \\
\frac{d a(t)}{d t}=\alpha i+\beta_{3} i a-\mu a .
\end{gathered}
$$

Theorem 1. System (3) has no cycle.

Proof. Leting $G=1 /\left(\alpha i+\beta_{3} i a\right)$, we denote the right sides of system (3) by $f_{1}$ and $f_{2}$, respectively, and it follows that

$$
\frac{\partial\left(G f_{1}\right)}{\partial i}+\frac{\partial\left(G f_{2}\right)}{\partial a}
$$




$$
\begin{aligned}
&=\frac{1}{\left(\alpha i+\beta_{3} a i\right)^{2}}\{-\left[\beta_{1} i^{2}+\left(p+\beta_{2} a\right)(1-a)\right] \\
&\left.\times\left(\alpha+\beta_{3} a\right)-\mu \alpha i\right\}<0 .
\end{aligned}
$$

So, Dulac's criteria imply that system (3) does not have a limit cycle [13].

As we all know, interpersonal communication is considered more effective in influencing an individual's decision to adopt [12]. For investigating the persuasive advertisement's effect in the decision-making stage, we will analyze the dynamics of system (3) in two kinds of parameter assumptions: in the decision-making process, I: $\alpha=0$, the persuasive advertisement's effect is neglected, and II: $\alpha \neq 0$, the persuasive advertisement's effect is considered. We first discuss system (3) under the assumption Case I.

Case I $(\alpha=0)$. When $\alpha=0$, we can derive that system (3) has the equilibrium $P^{0}\left(i^{0}, 0\right)$, where $i^{0}=\left(1 / 2 \beta_{1}\right)\left(\beta_{1}-p-\right.$ $\gamma+\sqrt{\left.\left(p+\gamma-\beta_{1}\right)^{2}+4 p \beta_{1}\right)}<1$. For considering its local stability, we first give that the jacobian matrix of system (3) at the generic equilibrium is

$$
J=\left(\begin{array}{cc}
\beta_{1}(1-i-a)-\left(p+\beta_{1} i+\beta_{2} a\right)-\gamma-\beta_{3} a & \beta_{2}(1-i-a)-\left(p+\beta_{1} i+\beta_{2} a\right)-\beta_{3} i \\
\beta_{3} a & \beta_{3} i-\mu
\end{array}\right) .
$$

Furthermore,

$$
\left.J\right|_{P^{0}}=\left(\begin{array}{cc}
\beta_{1}\left(1-i^{0}\right)-\left(p+\beta_{1} i^{0}+\gamma\right) & \beta_{2}\left(1-i^{0}\right)-\left(p+\beta_{1} i^{0}+\beta_{3} i^{0}\right) \\
0 & \beta_{3} i^{0}-\mu
\end{array}\right),
$$

and we assume that its character roots are $\lambda_{1}$ and $\lambda_{2}$; then $\lambda_{1}=\beta_{1}\left(1-i^{0}\right)-\left(p+\beta_{1} i^{0}+\gamma\right)=-p / i^{0}-\beta_{1} i^{0}<0, \lambda_{2}=$ $\beta_{3} i^{0}-\mu$. Define the threshold $R \triangleq \beta_{3} i^{0} / \mu$; the result as follows is obtained.

Theorem 2. System (3) always has a trivial equilibrium $P^{0}\left(i^{0}, 0\right)$; if $R<1, P^{0}$ is locally asymptotically stable; furthermore, it is globally asymptotically stable; if $R>1, P^{0}$ is unstable.

Remark 3. In the early stage of new products diffusion, there are some people who have been aware of the information about the product through mass media in the awareness stage but have not yet adopted it, and at this moment, the fraction of population $U$ is $1-i^{0}$, the fraction of population $I$ is $i^{0}$, and then $R$ is the average number (related to the number of population $I$ at the initial time) of new adopters caused by a single adopter introduced into population exclusive adopters in the early stage of the diffusion.

Next, we consider the existence and stability of the positive equilibrium. Letting the right side of equations in system (3) equal to zero, we obtain $i^{*}=\mu / \beta_{3}$, and

$$
\begin{aligned}
F_{1}(a) \triangleq \beta_{2} a^{2} & +\left[p-\beta_{2}+\left(\beta_{1}+\beta_{2}+\beta_{3}\right) \frac{\mu}{\beta_{3}}\right] a \\
& +\beta_{1}\left(\frac{\mu}{\beta_{3}}\right)^{2}+\left(p+\gamma-\beta_{1}\right) \frac{\mu}{\beta_{3}}-p=0 .
\end{aligned}
$$

When $R>1, i^{*}=\mu / \beta_{3}<i^{0}<1$; notice that $\beta_{1}\left(i^{0}\right)^{2}+(p-$ $\left.\beta_{1}+\gamma\right) i^{0}-p=0$, so $\beta_{1}\left(\mu / \beta_{3}\right)^{2}+\left(p+\gamma-\beta_{1}\right)\left(\mu / \beta_{3}\right)-p<0$, so (7) has a unique solution $a^{*}$ in $(0,1)$; when $R<1$, (7) has no positive solution.

Theorem 4. When $R>1$, system (3) has the equilibrium $P^{0}$ and $P^{*}\left(\mu / \beta_{3}, a^{*}\right), P^{0}$ is unstable, and $P^{*}$ is globally asymptotically stable.

Remark 5. When $\beta_{3}>\mu / i^{0}>\mu$, new products can still diffuse successfully even if the influence of mass media is neglected in the decision-making stage.

Case II $(\alpha \neq 0)$. When $\alpha \neq 0$, let

$$
\begin{gathered}
\left(p+\beta_{1} i+\beta_{2} a\right)(1-i-a)-\gamma i-\alpha i-\beta_{3} i a=0, \\
\alpha i+\beta_{3} i a-\mu a=0 .
\end{gathered}
$$

From the second equation of the formula (8), we derive $\hat{i}=$ $\mu a /\left(\alpha+\beta_{3} a\right)<\mu / \beta_{3}$, so, we assume $\mu<\beta_{3}<1$ to guarantee the positive equilibrium's existence. Substituting $\hat{i}$ into the first equation of the formula (8), we obtain

$$
F_{2}(a) \triangleq q_{0} a^{4}+q_{1} a^{3}+q_{2} a^{2}+q_{3} a+q_{4}=0 .
$$

Here $q_{0}=\beta_{2} \beta_{3}^{2}>0$,

$$
\begin{aligned}
& q_{1}=2 \alpha \beta_{2} \beta_{3}+\left(p-\beta_{2}\right) \beta_{3}^{2}+\mu\left(\beta_{1}+\beta_{2}+\beta_{3}\right) \beta_{3}>0, \\
& q_{2}=\alpha^{2} \beta_{2}+2 \alpha\left(p-\beta_{2}\right) \beta_{3}+\alpha \mu\left(\beta_{1}+\beta_{2}+\beta_{3}\right)+\mu^{2} \beta_{1}+ \\
& \mu\left(p+\alpha+\gamma-\beta_{1}\right) \beta_{3}-p \beta_{3}^{2}, \\
& q_{3}=\left(p-\beta_{2}\right) \alpha^{2}+\mu\left(p+\alpha+\gamma-\beta_{1}\right) \alpha-2 p \alpha \beta_{3}, \\
& q_{4}=-p \alpha^{2}<0 .
\end{aligned}
$$




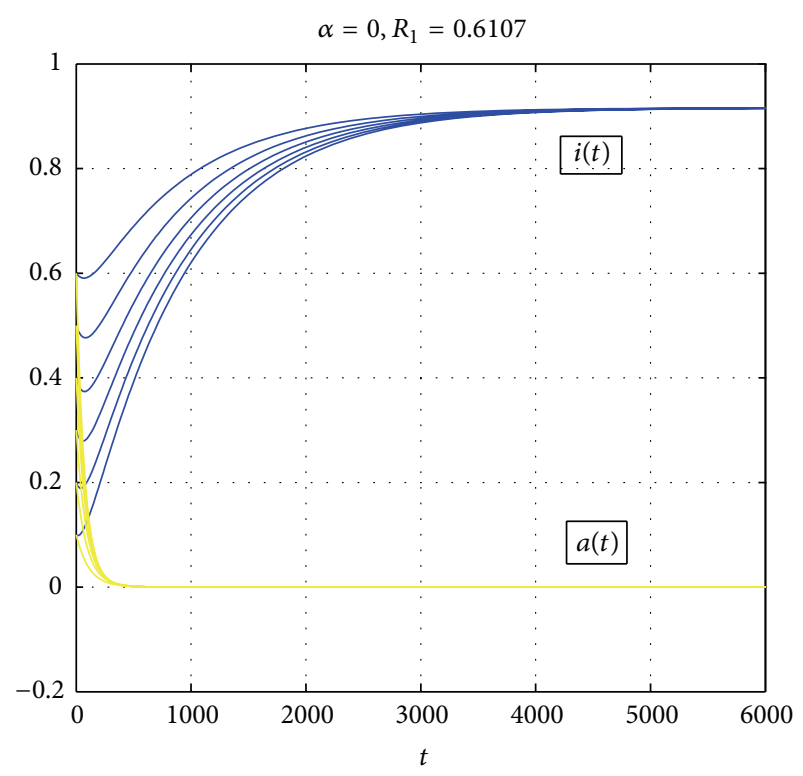

(a)

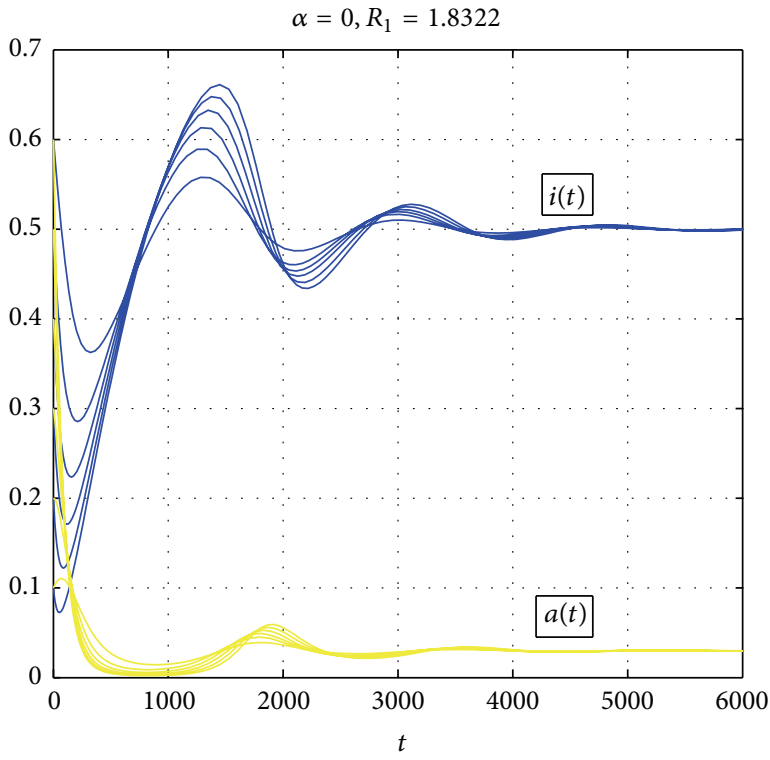

(b)

Figure 1: (a) and (b) are time series of system (3) when $\alpha=0$.

Notice that $F_{2}(0)<0, F_{2}(1)=q_{0}+q_{1}+q_{2}+q_{3}+q_{4}=$ $\mu\left(\alpha+\beta_{3}\right)\left(\beta_{2}+\beta_{3}+p+\alpha+\gamma\right)+\mu^{2} \beta_{1}>0$, so (9) at least has one positive solution in the interval $(0,1)$, and

$$
\begin{aligned}
& F_{2}^{\prime}(a)=4 q_{0} a^{3}+3 q_{1} a^{2}+2 q_{2} a+q_{3}, \\
& F_{2}^{\prime \prime}(a)=12 q_{0} a^{2}+6 q_{1} a+2 q_{2}, \\
& F_{2}^{\prime \prime}(1)=5 \beta_{2} \beta_{3}^{2}+6 \alpha \beta_{2} \beta_{3}+2\left(p-\beta_{2}\right)\left(\beta_{3}^{2}+\alpha \beta_{3}\right)+\mu\left(\beta_{1}+\right. \\
& \left.\beta_{2}+\beta_{3}\right)\left(\alpha+\beta_{3}\right)+\alpha^{2} \beta_{2}+\mu^{2} \beta_{1}+\mu\left(p+\alpha+\gamma-\beta_{1}\right) \beta_{3}>0 .
\end{aligned}
$$

When $q_{2} \geq 0$, because $q_{1}>0, F_{2}^{\prime \prime}(a)=0$ has no positive root on $(0,1)$ and $F_{2}^{\prime \prime}(1)>0$, this means that $F_{2}(a)$ is concave on $[0,1]$; moreover $F_{2}(a)$ either increases on $[0,1]$ or decreases first and then increases on $[0,1]$, and it follows that (9) has a unique root on $(0,1)$.

When $q_{2}<0$, on one hand, we can deduce that $F_{2}^{\prime \prime}(a)=$ 0 has a positive root on $(0,1)$ which means that $F_{2}(a)$ has inflection points on $(0,1)$. Combining $F_{2}^{\prime \prime}(1)>0$, it is proved that $F_{2}(a)$ is convex first and then concave on $(0,1)$. On the other hand, we can also deduce that $F_{2}^{\prime}(0)<0$, which show that (9) has a unique root on $(0,1)$.

In summary, (9) has a unique root $\widehat{a}$ on $(0,1)$; therefore, we have the following result.

Theorem 6. When $\beta_{3}>\mu$, system (3) has a unique positive equilibrium $\widehat{P}(\hat{i}, \widehat{a})$, and it is globally asymptotically stable.

Remark 7. New products diffusion is successful when $\beta_{3}>\mu$, and it is obvious that the critical value of successful diffusion decrease when $\alpha \neq 0$, which means new products' successful diffusion is relatively easier than which there is no persuasive advertisement's effect in the decision-making stage.

\section{Numerical Simulation}

To verify our analysis, we give the simulation results. Figure 1 is time series of system (3) with different initial values; parameters are chosen as $p=0.001, \beta_{1}=0.0001, \beta_{2}=$ $0.0004, \gamma=0.0001$, and $\mu=0.015$. In Figure 1 , we fix $u(0)=0.3$; by $i(0)+a(0)=1-u(0)$, we take the initial value $(i(0), a(0))$ as $(0.6,0.1),(0.3,0.4),(0.1,0.6),(0.2,0.5)$, $(0.4,0.3)$, and $(0.5,0.2)$, respectively. (a) Figure 1 suggests that the equilibrium $P^{0}$ is globally asymptotic stable when $\alpha=$ $0, \beta_{3}=0.01$, and $R=0.6107$. Namely, when $R<1$, although there are many people who are aware of the product, no one buys it, and new products cannot diffuse on the market; (b) suggests the equilibrium $P^{*}$ is globally asymptotically stable when $\alpha=0, \beta_{3}=0.03$, and $R=1.8322$; it shows that new products can diffuse successfully on the market when $R>1$.

\section{Sensitivity Analysis and Diffusion Strategies}

In real world applications, our main objective is to guarantee that new products can diffuse on the market. So, we consider the influence of the parameter of the system on products diffusion; that is to say, we study the relationship between the basic reproduction number $R$ and parameters in the model. By some calculations, it is obvious that

$$
\begin{aligned}
& \frac{\partial R}{\partial p}=\frac{\beta_{3}}{2 \mu \beta_{1}}\left(-1+\frac{p+\gamma+\beta_{1}}{\sqrt{\left(p+\gamma-\beta_{1}\right)^{2}+4 p \beta_{1}}}\right)>0, \\
& \frac{\partial R}{\partial \beta_{1}}=\left(\beta _ { 3 } \left[(p+\gamma) \sqrt{\left(p+\gamma-\beta_{1}\right)^{2}+4 p \beta_{1}}\right.\right. \\
& \left.\left.-\left(\left(p+\gamma-\beta_{1}\right)(p+\gamma)+2 p \beta_{1}\right)\right]\right)
\end{aligned}
$$






$p=0.001$

$p=0.01$

$\longrightarrow p=0.1$

(a)

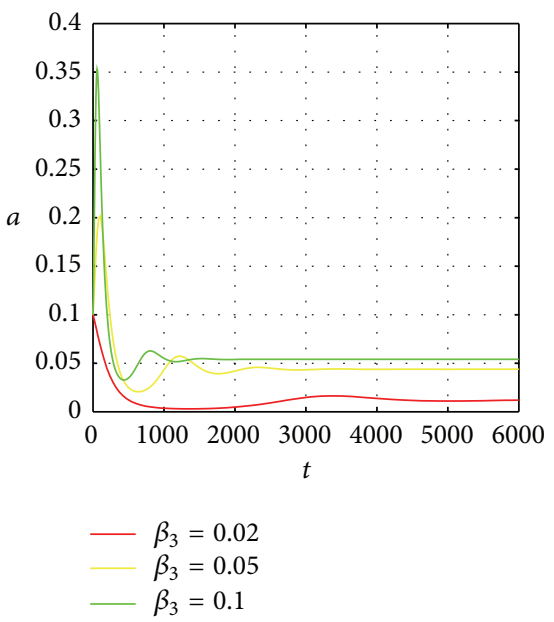

(d)
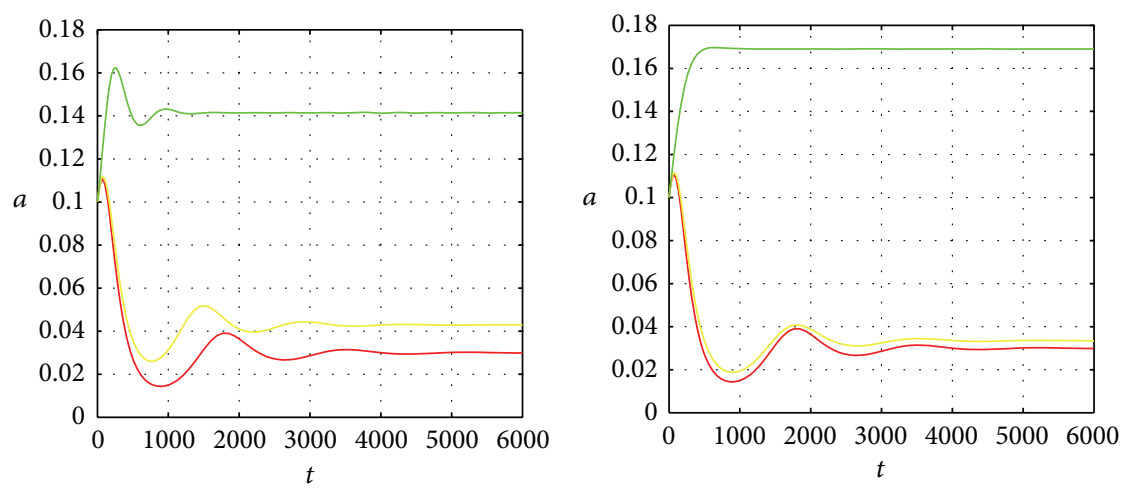

$\begin{aligned}-\beta_{1} & =0.0001 \\ \beta_{1} & =0.001 \\ -\beta_{1} & =0.01\end{aligned}$

(b)

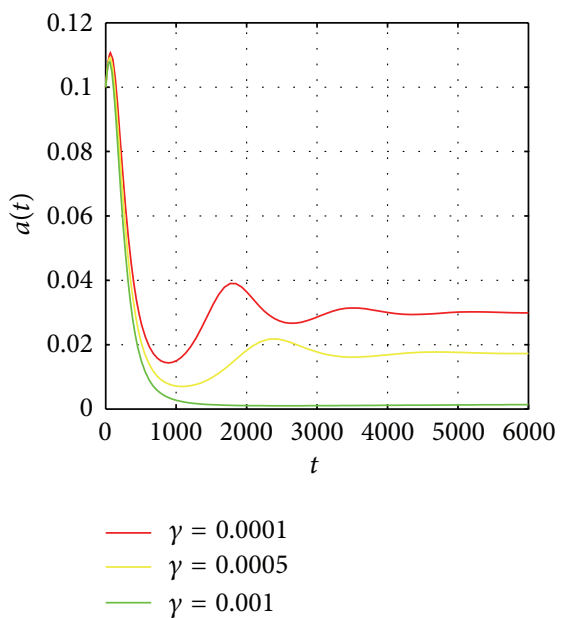

(e)

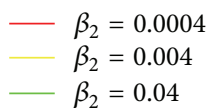

(c)

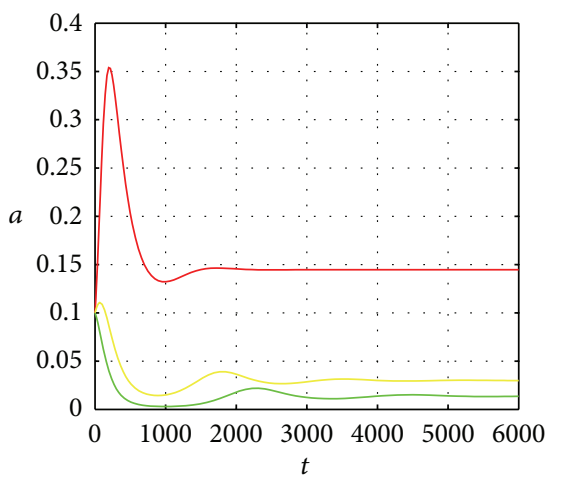

$\begin{aligned}-\mu & =0.005 \\ \mu & =0.015 \\ \mu & =0.02\end{aligned}$

(f)

FIGURE 2: When $\alpha=0$, evolution of $a(t)$ for different values of $p, \beta_{1}, \beta_{2}, \beta_{3}, \gamma$, and $\mu$, respectively.

$$
\begin{gathered}
\times\left(2 \mu \beta_{1}^{2} \sqrt{\left(p+\gamma-\beta_{1}\right)^{2}+4 p \beta_{1}}\right)^{-1}>0 \\
\frac{\partial R}{\partial \beta_{3}}=\frac{\beta_{1}-p-\gamma+\sqrt{\left(p+\gamma-\beta_{1}\right)^{2}+4 p \beta_{1}}}{2 \mu \beta_{1}}>0,
\end{gathered}
$$

while

$$
\begin{gathered}
\frac{\partial R}{\partial \mu}=-\frac{\beta_{3}\left(\beta_{1}-p-\gamma+\sqrt{\left(p+\gamma-\beta_{1}\right)^{2}+4 p \beta_{1}}\right)}{2 \mu^{2} \beta_{1}}<0, \\
\frac{\partial R}{\partial \gamma}=\frac{\beta_{3}}{2 \mu \beta_{1}}\left(-1+\frac{p+\gamma-\beta_{1}}{\sqrt{\left(p+\gamma-\beta_{1}\right)^{2}+4 p \beta_{1}}}\right)<0 .
\end{gathered}
$$

It can be seen that, among these parameters, $R$ is an increasing function of $p, \beta_{1}$, and $\beta_{3}$. Opposed to this, $\mu$ and $\gamma$ have an inversely proportional relationship with $R$. So, in order to guarantee new products can diffuse on the market, namely, to make $R$ larger than one, we can take some measures such as enhancing the effect of mass media in the awareness stage and reinforcing the interpersonal persuasion in the decisionmaking stage and so forth.

It is not enough that new products can diffuse on the market; we are more willing to see that there are more and more adopters. For this purpose, we will show the influences of parameters on the population $a(t)$ in Figures 2 and 3. It is observed that the peak and the stable state of $a(t)$ increase while $p, \beta_{1}, \beta_{2}$, and $\beta_{3}$ are increasing, respectively, or while $\mu$ and $\gamma$ is declining, respectively, and that the stable state is arrived in advance, while the peak is postponed. The peak and the stable state of $a(t)$ do not change clearly when $\beta_{1}, \beta_{2}, \beta_{3}$, or $\gamma$ varies respectively. When $\beta_{3}$ is increasing, the stable state of $a(t)$ has no obvious variation, but its peak obviously increases.

Figure 3 is time series of system (3) when $\alpha=0, \alpha=$ $0.0005, \alpha=0.005, \alpha=0.05$, and $\beta_{3}=0.03$. We find that with the increment of $\alpha$, the density of adopters $a$ gradually 


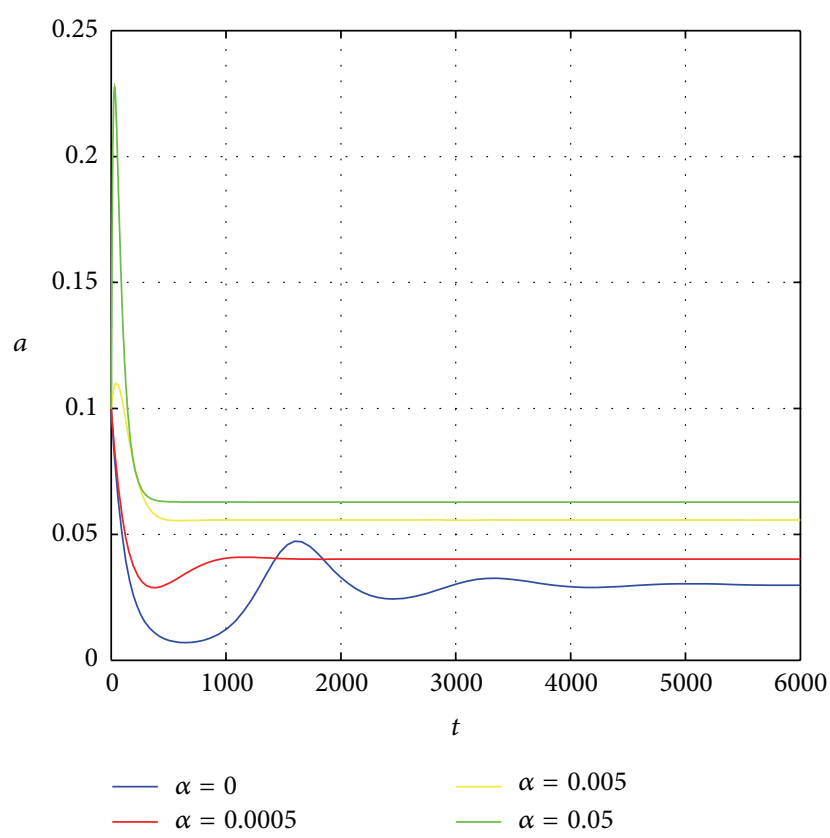

FIgURE 3: Time series of $a(t)$ with changes of $\alpha$.

increases, and the speed tending to steady states becomes more faster.

To sum up, enhancing the effect of mass media in two stages, reinforcing the interpersonal persuasion in the decision-making stage, and decreasing the loss of adopters are effective measures to new products diffusion with the number of adopters increasing and the arrival time of the stable state decreasing.

\section{Conclusion and Discussion}

In this paper, we have proposed a homogeneous model with the awareness stage and the decision-making stage to expound new products diffusion processes. We assume product information spreads due to two routes: word-ofmouth among people's direct contacts and mass media channels. If the influence of mass media is neglected in the decision-making stage, we have shown that the model admits a threshold $R$. when $R<1$, namely, $\beta_{3}<\mu / i^{0}$, new products diffusion is unsuccessful, few people being aware of the product and no one buying it; when $R>1$, namely, $\beta_{3}>\mu / i^{0}>\mu$, new products diffusion is successful, which means new products can diffuse on the market even without the influence of mass media in the decision-making stage. If the influence of mass media is considered in the decisionmaking stage, when $\beta_{3}>\mu$, new products diffusion is successful. It is obvious that the critical value of successful diffusion decreases. All theoretical results are confirmed by numerical simulations, and some diffusion strategies are given by parameters' sensitive analysis.

Simulations imply that persuasive advertisement is very helpful to the prevalence of new products from the perspective of economics, and it can accelerate new products prevalence. In addition, simulations also indicate that enterprises should take different advertisement strategies in different stages: before adopters tend to stable states, enterprises should take the persuasive advertisement strategy to tell consumers why they should choose a particular brand; after adopters tend to stable states, namely, once the brand is popularized, the enterprise should take the remindful advertisement strategy to remind consumers that there is such a product, which is useful to save advertising cost.

\section{Acknowledgments}

This work is supported by a Grant from the National Natural Science Foundation of China (11171314, 11201434, and 11301491), TianYuan Special Foundation of the National Natural Science Foundation of China (11226259), Shanxi province Natural Science Foundation (2012011002-1 and 20130210021), and Scientific research item for the Returned Overseas Chinese Scholars of Shanxi province (2010-074).

\section{References}

[1] F. M. Bass, "A new product growth model for consumer durables," Management Science, vol. 15, pp. 215-227, 1969.

[2] V. Mahajan, E. Muller, and R. A. Kerin, "Introduction strategy for a new products with positive and negative word-of-mouth," Management Science, vol. 30, pp. 1389-1404, 1984.

[3] V. Mahajan and R. Peterson, Models for Innovation Diffusion, Sage, Beverly Hills, Calif, USA, 1985.

[4] Y. Yu and W. Wang, "Global stability of an innovation diffusion model for $n$ products," Applied Mathematics Letters, vol. 19, no. 11, pp. 1198-1201, 2006.

[5] W. Wendi, P. Fergola, and C. Tenneriello, "Innovation diffusion model in patch environment," Applied Mathematics and Computation, vol. 134, no. 1, pp. 51-67, 2003.

[6] H.-S. Yan and K.-P. Ma, "Competitive diffusion process of repurchased products in knowledgeable manufacturing," European Journal of Operational Research, vol. 208, no. 3, pp. 243252, 2011.

[7] Y. Yu, W. Wang, and Y. Zhang, "An innovation diffusion model for three competitive products," Computers \& Mathematics with Applications, vol. 46, no. 10-11, pp. 1473-1481, 2003.

[8] K. de Valck, G. H. van Bruggen, and B. Wierenga, "Virtual communities: a marketing perspective," Decision Support Systems, vol. 47, pp. 185-203, 2009.

[9] P. Gaudiano, O. Bandte, D. Duzevik, and D. Anev, "How word-of-mouth impacts medicare product launch and product design," in Proceedings of the Word of Mouth Marketing Summit (WOMMA '07), Las Vegas, Nev, USA, 2007.

[10] C. Kiss and M. Bichler, "Identification of influencers-measuring influence in customer networks," Decision Support Systems, vol. 46, pp. 233-253, 2008.

[11] W. Wang, P. Fergola, S. Lombardo, and G. Mulone, "Mathematical models of innovation diffusion with stage structure," Applied Mathematical Modelling, vol. 30, pp. 129-146, 2006.

[12] E. M. Rogers, Diffusion of Innovations, The Free Press, New York, NY, USA, 4th edition, 1995.

[13] L. Perko, Differential Equations and Dynamical Systems, vol. 7, Springer, New York, NY, USA, 2nd edition, 1996. 


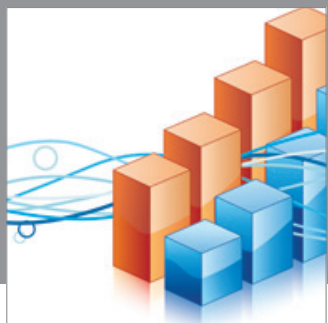

Advances in

Operations Research

mansans



The Scientific World Journal
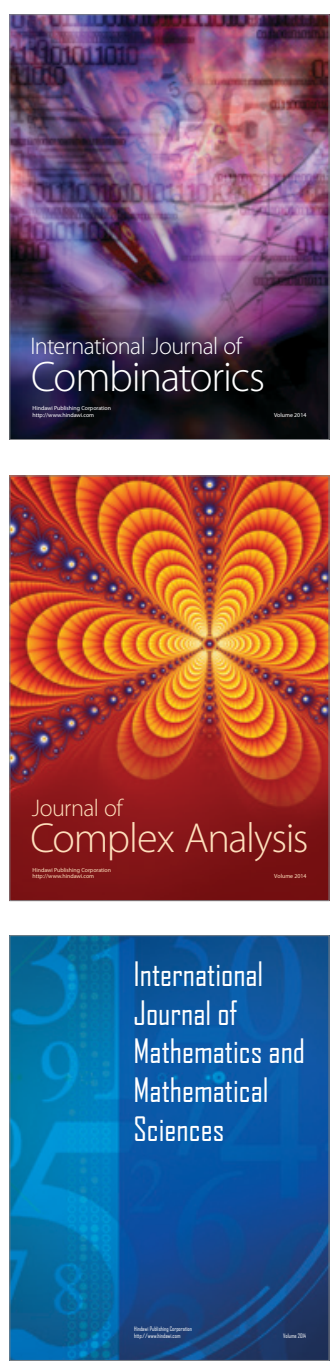
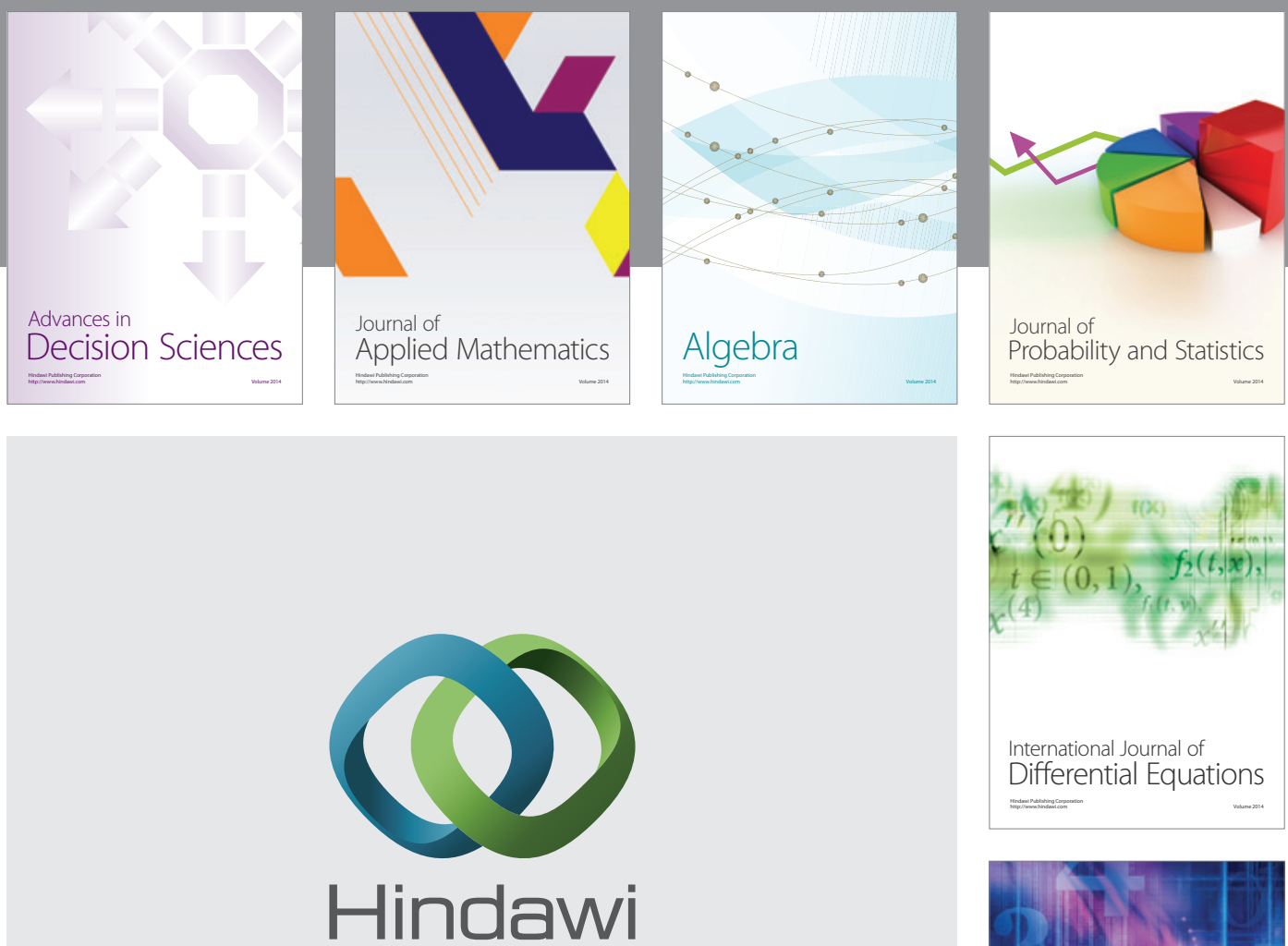

Submit your manuscripts at http://www.hindawi.com
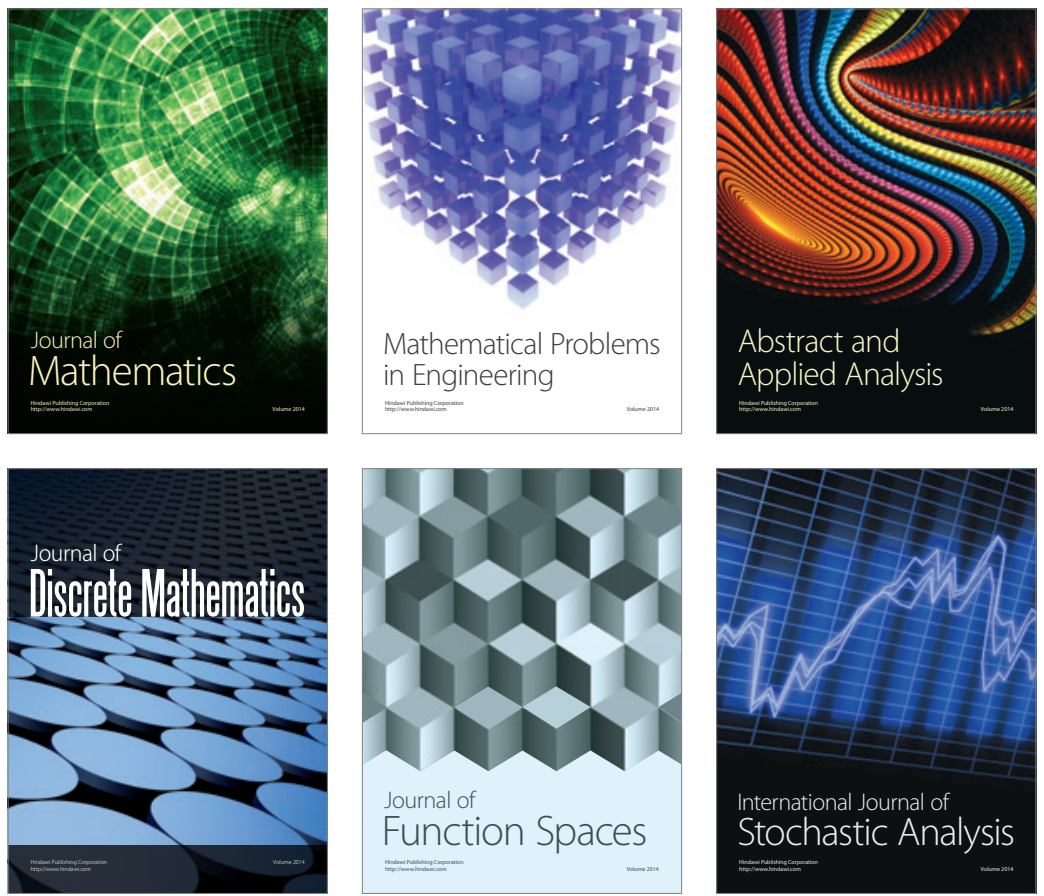

Journal of

Function Spaces

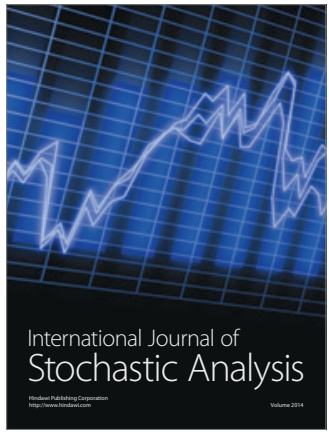

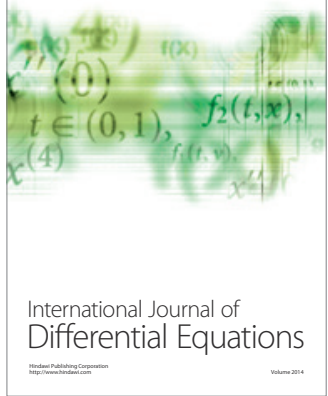
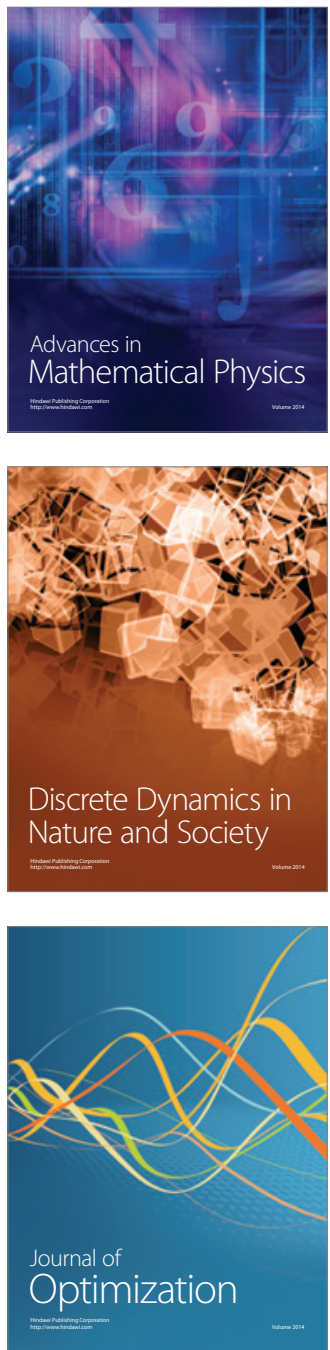